\title{
ELECTRON SCATTERING BY INTERFACE POLAR OPTICAL PHONONS IN DOUBLE BARRIER HETEROSTRUCTURES
}

\author{
J. Požela, K. Požela, and V. Jucienė \\ Semiconductor Physics Institute, A. Goštauto 11, LT-01108 Vilnius, Lithuania \\ E-mail:pozela@pfi.lt
}

Received 12 December 2006

\begin{abstract}
The confined electron-interface (IF) polar optical phonon scattering in double heterostructures is considered within the dielectric continuum approach. The dependences of electron-IF phonon scattering rate (SR) on the quantum well (QW) width and on the IF phonon frequencies are calculated. The intrasubband SR of electrons confined in the QW by IF phonons is estimated for AlAs / GaAs / AlAs, GaAs / InAs / GaAs, and GaN/InN/GaN heterostructures. The SR of electrons, the energy of which is higher than the barrier phonon energy, increases with an increase of the phonon energy. It is shown that the SR of electrons, the energy of which corresponds to the bulk phonon energy in a QW material, by symmetric IF phonons strongly decreases with a decrease of the QW width, when the width is smaller than 5-10 $\mathrm{nm}$. Contrary, the SR of electrons, the energy of which exceeds the highest IF phonon energy, by IF phonons increases in a narrow QW. This means that the electron mobility and the saturated drift velocity at high electric fields in a narrow QW must be higher than in a wide one.
\end{abstract}

Keywords: electron-phonon scattering, quantum wells, heterojunctions

PACS: 72.10.Di, 73.21.Fg, 73.40.Kp

\section{Introduction}

A decrease of inelastic electron-polar optical (PO) phonon scattering rate means an increase of the electron maximum drift velocity [1]. The electron maximum drift velocity determines the main parameters of transistors, namely the cutoff frequency and gain. An essential decrease of the scattering rate (SR) of the electrons confined in a quantum well $(\mathrm{QW})$ by the $\mathrm{PO}$ phonons confined in a phonon well with a decrease of the well width was reported in several papers [1-3]. However, the calculations [4-6] show that this decrease of the electron-confined phonon SR is compensated by an equivalent increase of the electron-interface (IF) phonon SR.

In this paper, a possibility to reduce the electron-IF phonon SR by choosing a relevant pair of semiconductors in a double heterostructure and by making use of a variation of IF phonon frequency in narrow QWs is considered.

\section{PO phonon potential amplitudes and envelope functions in a quantum well between two phonon barriers}

Let us, following the dielectric continuum model [3-5], describe the PO phonon envelope function and the potential amplitude in a double heterojunction structure taking into account the dependence of IF phonon frequencies on the QW width.

Figure 1 illustrates the considered structure. Electrons are confined in the electron $\mathrm{QW}$, located in the

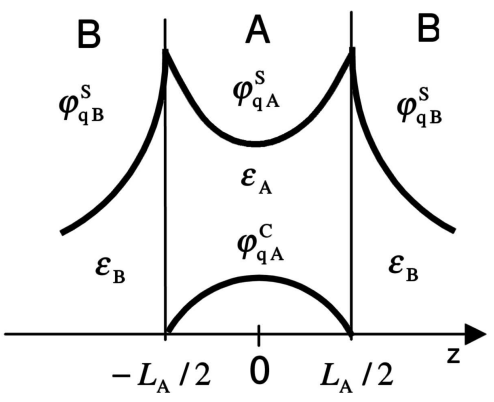

Fig. 1. Schematic view of the layered heterostructure under study. Electrons and phonons are confined in the layer $A$ between the barriers $B . \varphi_{\mathbf{q}}^{\mathrm{C}}$ and $\varphi_{\mathbf{q}}^{\mathrm{S}}$ illustrate the confined and symmetric IF phonon envelope functions. $L_{A}$ is the $\mathrm{QW}$ width. 
material $A$ between the potential barriers $B$. Due to a difference of the longitudinal PO phonon frequencies in the $\mathrm{QW}$ material $A, \omega_{\mathrm{L} A}$, and the barrier material $B$, $\omega_{\mathrm{LB}}$, the optical phonons are confined in the QW.

The PO phonon potential $\varphi(\mathbf{q})$ in the $\mathrm{QW}$ is found from the solution of the Laplace's equation for a case of the QW (material $A$ ) between two phonon barriers (material $B$ ). The boundary conditions at the interfaces $\pm L_{A} / 2$ are:

$$
\begin{aligned}
\varphi_{A}(\mathbf{q}) & =\varphi_{B}(\mathbf{q}) \\
\varepsilon_{A}(\omega) \frac{\partial \varphi_{A}(\mathbf{q})}{\partial z} & =\varepsilon_{A}(\omega) \frac{\partial \varphi_{B}(\mathbf{q})}{\partial z} .
\end{aligned}
$$

Note that $\mathbf{q}$ is the phonon wave vector in the QW plane.

The QW and barrier dielectric functions for binary materials are:

$$
\begin{aligned}
& \varepsilon_{A}(\omega)=\varepsilon_{\infty A} \frac{\omega^{2}-\omega_{\mathrm{L} A}^{2}}{\omega^{2}-\omega_{\mathrm{T} A}^{2}}, \\
& \varepsilon_{B}(\omega)=\varepsilon_{\infty B} \frac{\omega^{2}-\omega_{\mathrm{L} B}^{2}}{\omega^{2}-\omega_{\mathrm{T} B}^{2}},
\end{aligned}
$$

where $\varepsilon_{\infty A, B}$ are the high-frequency dielectric permittivities, $\omega_{\mathrm{L} B}$ and $\omega_{\mathrm{T} B}$ are the longitudinal and transverse optical phonon frequencies in the barrier material $B$, and $\omega_{\mathrm{L} A}$ and $\omega_{\mathrm{T} A}$ are those in the phonon well.

Due to translational invariance in the direction parallel to the interface, the solutions can be presented in the form

$$
\varphi(\mathbf{q})=F(\mathbf{q}) \varphi_{\mathbf{q}}(z) \mathrm{e}^{\mathrm{i}\left(\mathbf{q r}_{\|}\right)},
$$

where $\varphi_{\mathbf{q}}(z)$ is the $z$-component of the phonon envelope function, $F(\mathbf{q})$ is the normalization coefficient (phonon potential amplitude), and $\mathbf{q}$ and $\mathbf{r}_{\|}$are the phonon wave vector and the coordinate in the interface plane $(x, y)$.

The solution of the Laplace's equation gives two different types of phonon modes in the phonon well $[4,5]$ : (i) the confined modes and (ii) the symmetric and antisymmetric IF modes.

The confined phonon envelope functions for the phonon well of the width $L_{A}$ have the form

$$
\varphi_{\mathbf{q}}^{\mathrm{C}}(z)=\left\{\begin{array}{l}
\cos \left(\frac{\nu \pi z}{L_{A}}\right), \nu=1,3,5, \ldots \\
\sin \left(\frac{\nu \pi z}{L_{A}}\right), \nu=2,4,6, \ldots
\end{array},\right.
$$

for $-L_{A} / 2<z<L_{A} / 2$.
The IF phonon envelope functions are determined by the expressions

$$
\begin{aligned}
& \varphi_{\mathbf{q}}^{\mathrm{S}}(z)= \exp (+\mathbf{q} z) \exp \left(+\mathbf{q} \frac{L_{A}}{2}\right), z \leq-\frac{L_{A}}{2} \\
& \varphi_{\mathbf{q}}^{\mathrm{S}}(z)=\frac{\exp (+\mathbf{q} z)+\exp (-\mathbf{q} z)}{\exp \left(+\mathbf{q} \frac{L_{A}}{2}\right)+\exp \left(-\mathbf{q} \frac{L_{A}}{2}\right)} \\
& \quad-\frac{L_{A}}{2}<z<\frac{L_{A}}{2} \\
& \varphi_{\mathbf{q}}^{\mathrm{S}}(z)=\exp (-\mathbf{q} z) \exp \left(+\mathbf{q} \frac{L_{A}}{2}\right), z \geq \frac{L_{A}}{2}
\end{aligned}
$$

for the symmetric IF phonon modes, and by the expressions

$$
\begin{aligned}
& \varphi_{\mathbf{q}}^{\mathrm{A}}(z)=-\exp (+\mathbf{q} z) \exp \left(+\mathbf{q} \frac{L_{A}}{2}\right), z \leq-\frac{L_{A}}{2}, \\
& \varphi_{\mathbf{q}}^{\mathrm{A}}(z)=\frac{\exp (+\mathbf{q} z)-\exp (-\mathbf{q} z)}{\exp \left(+\mathbf{q} \frac{L_{A}}{2}\right)-\exp \left(-\mathbf{q} \frac{L_{A}}{2}\right)}, \\
& \quad-\frac{L_{A}}{2}<z<\frac{L_{A}}{2}, \\
& \varphi_{\mathbf{q}}^{\mathrm{A}}(z)=\exp (-\mathbf{q} z) \exp \left(+\mathbf{q} \frac{L_{A}}{2}\right), z \geq \frac{L_{A}}{2}
\end{aligned}
$$

for the antisymmetric ones.

Expressions (3)-(6) for the IF PO phonon potentials, which are obtained on the basis of the dielectric continuum (DC) model, are in good agreements with the IF phonon potentials calculated within the ab initio microscopic model $[5,7]$. It is worth to note a good agreement between the DC model-based calculated results and the experimentally observed Raman spectra caused by IF PO phonons $[8,9]$. The existence of IF phonons manifests itself in many experiments [9]. The electronIF phonon scattering is a dominant mechanism of electron scattering in narrow QWs [9-11].

It should be noted that expression (4) for the confined PO phonon envelope function does not coincide with the results of calculations within the microscopic model $[5,7]$. In the DC model, the Maxwell's conditions at an interface are satisfied and the mechanical ones are neglected. The problem of the boundary conditions for the confined PO phonons is discussed by many authors (see $[9,12]$ and references therein).

Huang and Zhu [13] have proposed an expression for the confined phonon potentials that satisfies both the mechanical $\left(\mathrm{d} \varphi_{q} / \mathrm{d} z=0\right)$ and Maxwell's conditions 
$\left(\varphi_{q}=0\right)$ at the interfaces. The resulting phonon potential function is in good agreement with calculations based on the microscopic model [7,9]. The DC model with the Huang-Zhu potential for confined phonons gives the best explanation of the experimental data on the electron-PO phonon interaction in ultrathin multiple QWs [10]. The calculations of QW width dependences of the electron-confined phonon SR in the AlAs / GaAs / AlAs QWs, using the Huang-Zhu and the $\varphi_{\mathbf{q}}^{\mathrm{C}}(z) \sim \cos q_{z} z$ (Eq. (4)) phonon potentials, yield the same results for QW widths less than 10-15 nm [14]. Therefore, in the calculations of the electronconfined phonon SR contribution we shall use the function (4) as an admissible approximation.

In the DC model, the phonon potential amplitude $F(\mathbf{q})$, which determines the electron-phonon interaction strength for the phonon well (material $A$ ) between the single phonon barriers (material $B$ ), is determined as in [3-5],

$$
F(\mathbf{q})=\left[S\left(\frac{\mathrm{d} \varepsilon_{A}(\omega)}{\mathrm{d} \omega} I_{A}+\frac{\mathrm{d} \varepsilon_{B}(\omega)}{\mathrm{d} \omega} I_{B}\right)\right]^{-1 / 2},
$$

where $S$ is the in-plane normalization area, and the parameters $I_{A}$ and $I_{B}$ are given by the expression

$$
I_{A(B)}=\int_{A(B)}\left[q^{2}\left|\varphi_{\mathbf{q}}^{A(B)}(z)\right|^{2}+\left|\frac{\mathrm{d} \varphi_{\mathbf{q}}^{A(B)}(z)}{\mathrm{d} z}\right|^{2}\right] \mathrm{d} z \text {. }
$$

Making use of the dielectric function expressions (2) one obtains

$$
\begin{aligned}
F(\mathbf{q})=\sqrt{\frac{\hbar}{S}} & {\left[\varepsilon_{\infty A} \frac{2 \omega_{\nu}\left(\omega_{\mathrm{L} A}^{2}-\omega_{\mathrm{T} A}^{2}\right)}{\left(\omega_{\nu}^{2}-\omega_{\mathrm{T} A}^{2}\right)^{2}} I_{A}\right.} \\
& \left.+\varepsilon_{\infty B} \frac{2 \omega_{\nu}\left(\omega_{\mathrm{L} B}^{2}-\omega_{\mathrm{T} B}^{2}\right)}{\left(\omega_{\nu}^{2}-\omega_{\mathrm{T} B}^{2}\right)^{2}} I_{B}\right]^{-1 / 2},
\end{aligned}
$$

where $\omega_{\nu}$ is the frequency of IF phonons. Note that $\mathbf{q}$ is the emitted (or absorbed) phonon momentum.

Equation (7), for the bulk phonons $\left(\omega=\omega_{\mathrm{L} A}\right.$ and $\left.\varphi_{\mathbf{q}}(z) \sim \exp (\mathrm{iq} z)\right)$ reduces to the widely used formula

$$
\begin{aligned}
& F_{\mathrm{B}}^{2}(\mathbf{q})=\frac{F\left(\omega_{\mathrm{L} A}\right)}{q^{2} V}, \\
& F\left(\omega_{\mathrm{L} A}\right)=\frac{\hbar \omega_{\mathrm{L} A}}{2}\left(\frac{1}{\varepsilon_{\infty}}-\frac{1}{\varepsilon_{\mathrm{S}}}\right), \varepsilon_{\mathrm{S}}=\frac{\omega_{\mathrm{L} A}^{2}}{\omega_{\mathrm{T} A}^{2}} \varepsilon_{\infty},
\end{aligned}
$$

where $V$ is the normalization volume, $\hbar \omega_{\mathrm{L} A}$ is the bulk PO phonon energy, and $F\left(\omega_{\mathrm{L} A}\right)$ corresponds to the Fröhlich constant of the electron-PO phonon interaction.
For the confined phonons in layer $A\left(\varepsilon_{A}=0\right.$ and $\omega=\omega_{\mathrm{L} A}$ ) Eq. (7) gives

$$
\begin{aligned}
& F_{\mathrm{C}}^{2}(\mathbf{q})=F\left(\omega_{\mathrm{L} A}\right)\left[\frac{\left(q^{2}+q_{z}^{2}\right) L_{A}}{2}\right]^{-1} S^{-1}, \\
& q_{z}=\left(\frac{\nu \pi}{L_{A}}\right)^{2}, \quad \nu=1,2,3, \ldots
\end{aligned}
$$

For the IF phonons we obtain

$$
\begin{aligned}
& F_{\mathrm{IF}}^{\mathrm{S}}\left(\omega_{\nu}\right)=\sqrt{\frac{\hbar}{S}}\left[\varepsilon_{\infty A} \frac{2 \omega_{\nu}\left(\omega_{\mathrm{L} A}^{2}-\omega_{\mathrm{T} A}^{2}\right)}{\left(\omega_{\nu}^{2}-\omega_{\mathrm{T} A}^{2}\right)^{2}} 2 \mathbf{q}\right. \\
& \left.\times \tanh \left(\mathbf{q} \frac{L_{A}}{2}\right)+\varepsilon_{\infty B} \frac{2 \omega_{\nu}\left(\omega_{\mathrm{L} B}^{2}-\omega_{\mathrm{T} B}^{2}\right)}{\left(\omega_{\nu}^{2}-\omega_{\mathrm{T} B}^{2}\right)^{2}} 2 \mathbf{q}\right]^{-1 / 2}
\end{aligned}
$$

for the symmetric modes and

$$
\begin{aligned}
& F_{\mathrm{IF}}^{\mathrm{A}}\left(\omega_{\nu}\right)=\sqrt{\frac{\hbar}{S}}\left[\varepsilon_{\infty A} \frac{2 \omega_{\nu}\left(\omega_{\mathrm{L} A}^{2}-\omega_{\mathrm{T} A}^{2}\right)}{\left(\omega_{\nu}^{2}-\omega_{\mathrm{T} A}^{2}\right)^{2}} 2 \mathbf{q}\right. \\
& \left.\times \operatorname{coth}\left(\mathbf{q} \frac{L_{A}}{2}\right)+\varepsilon_{\infty B} \frac{2 \omega_{\nu}\left(\omega_{\mathrm{L} B}^{2}-\omega_{\mathrm{T} B}^{2}\right)}{\left(\omega_{\nu}^{2}-\omega_{\mathrm{T} B}^{2}\right)^{2}} 2 \mathbf{q}\right]^{-1 / 2}
\end{aligned}
$$

for the antisymmetric IF modes.

The IF phonon amplitude and, consequently, the strength of the electron-IF phonon interaction, according to Eqs. (11) and (12), is rather sensitive to the IF phonon frequencies $\omega_{\nu}$.

The frequencies $\omega_{\nu}$ of the IF phonon in the binary material phonon well of the width $L_{A}$ are found from the boundary conditions (1) [4],

$\varepsilon_{\infty} A \frac{\omega_{\mathrm{S}}^{2}-\omega_{\mathrm{L} A}^{2}}{\omega_{\mathrm{S}}^{2}-\omega_{\mathrm{T} A}^{2}} \tanh \left(\mathbf{q} \frac{L_{A}}{2}\right)+\varepsilon_{\infty B} \frac{\omega_{\mathrm{S}}^{2}-\omega_{\mathrm{L} B}^{2}}{\omega_{\mathrm{S}}^{2}-\omega_{\mathrm{T} B}^{2}}=0$

for the symmetric modes $\left(\omega_{\nu}=\omega_{\mathrm{S}}\right)$ and

$\varepsilon_{\infty A} \frac{\omega_{\mathrm{A}}^{2}-\omega_{\mathrm{L} A}^{2}}{\omega_{\mathrm{A}}^{2}-\omega_{\mathrm{T} A}^{2}} \operatorname{coth}\left(\mathbf{q} \frac{L_{A}}{2}\right)+\varepsilon_{\infty B} \frac{\omega_{\mathrm{A}}^{2}-\omega_{\mathrm{L} B}^{2}}{\omega_{\mathrm{A}}^{2}-\omega_{\mathrm{T} B}^{2}}=0$

for the antisymmetric $\left(\omega_{\nu}=\omega_{\mathrm{A}}\right)$ ones.

Figure 2 shows the dependences of $\omega_{S}$ on the width $L_{A}$ for the AlAs/GaAs/AlAs, GaAs/InAs/GaAs, and $\mathrm{GaN} / \mathrm{InN} / \mathrm{GaN}$ phonon wells at the fixed $q$ value $q=\sqrt{2 m \hbar \omega_{\mathrm{LA}} / \hbar^{2}}$. For GaAs, $q=\pi /(12.6 \mathrm{~nm})$.

The IF phonon frequencies are not equal to the frequency of the bulk phonons in the QW material. Moreover, there are two branches of symmetric and antisymmetric IF phonon frequencies: the barrier-like, $\omega_{B}$, and the QW-like, $\omega_{A}$. The barrier-like IF phonon frequencies are near to the barrier material optical phonon 


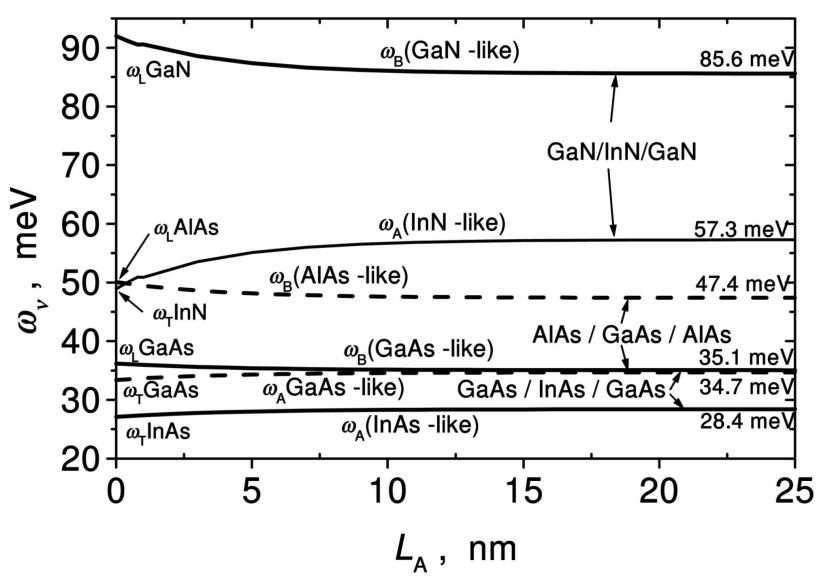

Fig. 2. The QW width dependences of the symmetric IF phonon frequencies for the $\mathrm{GaN} / \mathrm{InN} / \mathrm{GaN}$, AlAs/GaAs/AlAs, and $\mathrm{GaAs} / \mathrm{InAs} / \mathrm{GaAs}$ double heterostructures. $\omega_{A}$ and $\omega_{B}$ are the QW-like (material $A$ ) and the barrier-like (material $B$ ) frequencies, respectively. The values of the frequencies $\omega_{A}$ and $\omega_{B}$ at $L_{A}=25 \mathrm{~nm}$ are indicated by the numbers.

frequency (between $\omega_{\mathrm{L} B}$ and $\omega_{\mathrm{T} B}$ ). The QW-like phonon frequency is near to the QW material optical phonon frequency (between $\omega_{\mathrm{L} A}$ and $\omega_{\mathrm{T} A}$ ). In wide QWs, the symmetric and antisymmetric IF phonon branches degenerate, $\omega_{\mathrm{S}}=\omega_{\mathrm{A}}$. In a narrow $\mathrm{QW}$ of width $L_{A}<\pi / q$, the degeneracy of symmetric and antisymmetric phonon frequencies is lifted. At $L_{A} \rightarrow 0$, the symmetric mode frequency of the barrierlike phonons is $\omega_{\mathrm{L} B}$ and of the QW-like one it is $\omega_{\mathrm{T} A}$. For the antisymmetric mode, contrary, the barrier-like phonon frequency is $\omega_{\mathrm{T} B}$, and the QW-like one is $\omega_{\mathrm{L} A}$.

Note that the dielectric function in the QW and barriers has an opposite sign, $\varepsilon_{A}\left(\omega_{\mathrm{S}}\right) \varepsilon_{B}\left(\omega_{\mathrm{S}}\right)<0$, and at $\omega_{\mathrm{S}} \rightarrow \omega_{\mathrm{T} A}, \varepsilon_{A}\left(\omega_{\mathrm{T} A}\right) \rightarrow \infty$.

The change of the phonon frequencies at low QW widths influences significantly a contribution of the IF phonons to the electron-phonon SR.

\section{General expression for scattering rate}

We shall describe the electron SR in a QW as a probability to emit (or absorb) the PO phonon by a confined electron per time unit. The probability of electron transition from the initial state $\mathbf{k}_{i}$ to any of final states $\mathbf{k}_{f}$ by emission (or absorption) of the $\nu$-mode phonon in time unit is equal to

$$
\begin{aligned}
W_{\nu}\left(\mathbf{k}_{i}\right)= & \frac{2 \pi}{\hbar}\left(N_{q \nu} \pm \frac{1}{2}+\frac{1}{2}\right) \frac{S}{(2 \pi)^{2}} \\
& \times \int_{\mathbf{k}_{f}}\left|G_{z}\right|^{2} \delta_{\mathbf{k}_{i}, \mathbf{k}_{f} \mp \mathbf{q}} \delta\left(E_{i}-E_{f} \mp \hbar \omega_{\nu}\right) \mathrm{d} \mathbf{k}_{f},
\end{aligned}
$$

where $\mathbf{k}_{i}$ and $\mathbf{k}_{f}$ are the electron wave vectors in a QW plane, $E_{i}$ and $E_{f}$ are the initial and final electron energies, respectively, $\hbar \omega_{\nu}$ is the $\nu$-mode phonon energy,

$$
\begin{aligned}
& N_{q}\left(\omega_{\nu}\right)=\left[\exp \left(\frac{\hbar \omega_{\nu}}{k T}\right)-1\right]^{-1}, \\
& \left|G_{z}\right|^{2}=\left|\int_{-L_{A} / 2}^{L_{A} / 2} \varphi_{e i} e \varphi_{\nu}(\mathbf{q}) \varphi_{e f}^{*} \mathrm{~d} z\right|^{2}, \\
& \mathrm{~d} \mathbf{k}_{f}=k_{f} \mathrm{~d} k_{f} \mathrm{~d} \theta=\mathrm{d} k_{f}^{2} \frac{\mathrm{d} \theta}{2},
\end{aligned}
$$

$\delta_{\mathbf{k}_{i}, \mathbf{k}_{f} \mp \mathbf{q}}$ is the Kronecker delta, $\varphi_{e i}, \varphi_{e f}, \varphi_{\nu}(\mathbf{q})$ are the normalized electron and $\nu$-mode phonon wavefunctions in the QW of width $L_{A}$. Signs '+' and '-' correspond to a phonon emission and absorption, respectively.

For simplicity, we shall consider the intrasubband electron transition in the first electron subband of the rectangular electron QW of width $L_{A}$ with infinite barriers. Then the electron wavefunction has the form

$$
\varphi_{e}=\sqrt{\frac{2}{L_{A}}} \cos \left(\frac{\pi z}{L_{A}}\right) \text { for }-\frac{L_{A}}{2}<z<+\frac{L_{A}}{2},
$$

and

$$
\varphi_{e i} \varphi_{e f}^{*}=\frac{2}{L_{A}} \cos ^{2}\left(\frac{\pi z}{L_{A}}\right) .
$$

After integration of Eq. (15) for $k_{f}^{2}$, assuming $E_{i}=$ $\hbar^{2} k_{i}^{2} /(2 m)$ and taking into account the conservation of energy and momentum $\mathrm{k}$ in a $\mathrm{QW}$ plane, we obtain

$$
\begin{aligned}
& W_{\nu}\left(\mathbf{k}_{i}\right)=W_{0}\left(N_{q \nu} \pm \frac{1}{2}+\frac{1}{2}\right) \int_{0}^{2 \pi} \frac{\varepsilon_{0}}{\hbar} F^{2}\left(q_{0}\right) \\
& \times\left|\int_{-L_{A} / 2}^{+L_{A} / 2} \frac{2}{L_{A}} \cos ^{2}\left(\frac{\pi z}{L_{A}}\right) \varphi_{q_{0}}^{\nu}\left(q_{0}, z\right) \mathrm{d} z\right|^{2} \frac{\mathrm{d} \theta}{2},
\end{aligned}
$$

with $W_{0}=m e^{2} / \pi \hbar^{2} \varepsilon_{0}$. Here $\varepsilon_{0}$ is the electric constant $\varepsilon_{0}=8.85418 \cdot 10^{-14} \mathrm{~A} \mathrm{~s} \mathrm{~V}^{-1} \mathrm{~cm}^{-1}$.

For GaAs $W_{0}=5.0 \cdot 10^{7} \mathrm{~cm}^{-1}$.

In Eq. (17), the in-plane momentum of emitted (sign '-') or absorbed (sign ' + ') phonons is equal to

$$
\mathbf{q}_{0}=\sqrt{k_{\mathrm{opt}}^{2}(2 y \mp 1-2 \sqrt{y} \sqrt{y \mp 1} \cos \theta)}
$$

with

$$
y=\frac{E_{i}}{\hbar \omega_{\nu}}, \quad k_{\mathrm{opt}}^{2}=\hbar \omega_{\nu} \frac{2 m}{\hbar^{2}},
$$


where $k_{\text {opt }}$ is the wave number of electrons with the energy equal to the phonon energy $\hbar \omega_{\nu}$.

The electron SR in the QW of width $L_{A}$ by confined phonons, according to Eqs. (4), (10), and (17) is equal to

$$
\begin{aligned}
& W_{\mathrm{C}}\left(\mathbf{k}_{i}\right)=W_{0} F_{0}\left(N_{q}\left(\omega_{\mathrm{L} A}\right) \pm \frac{1}{2}+\frac{1}{2}\right) \frac{4}{L_{A}^{2}} \\
& \times\left\{\sum_{n=1,3, \ldots} \int_{0}^{2 \pi\left|\int_{-L_{A} / 2}^{+L_{A} / 2} \cos ^{2}\left(\frac{\pi z}{L_{A}}\right) \cos \left(n \frac{\pi z}{L_{A}}\right) \mathrm{d} z\right|^{2}} \frac{\mathrm{d} \theta}{2}\left[q_{0}^{2}(\theta)+\left(n \frac{\pi}{L_{A}}\right)^{2}\right] \frac{L_{A}}{2}\right. \\
& +\sum_{n=2,4, \ldots} \int_{0}^{2 \pi\left|\int_{-L_{A} / 2}^{+L_{A} / 2} \cos ^{2}\left(\frac{\pi z}{L_{A}}\right) \sin \left(n \frac{\pi z}{L_{A}}\right) \mathrm{d} z\right|^{2}} \frac{\mathrm{d} \theta}{2}\left[q_{0}^{2}(\theta)+\left(n \frac{\pi}{L_{A}}\right)^{2}\right] \frac{L_{A}}{2}
\end{aligned}
$$

with $F_{0}=\left(\varepsilon_{0} / \hbar\right) F\left(\omega_{\mathrm{L} A}\right)$, where $F\left(\omega_{\mathrm{L} A}\right)$ is determined by Eq. (9).

The electron intrasubband SR by symmetric IF phonons, according to Eqs. (5), (11), and (17), is equal to

$$
\begin{aligned}
& W_{\mathrm{IF}}^{\mathrm{S}}\left(\mathbf{k}_{i}\right)=W_{0}\left(N_{q}\left(\omega_{\mathrm{S}}\right) \pm \frac{1}{2}+\frac{1}{2}\right) \frac{2}{L_{A}} \\
& \times \int_{0}^{2 \pi\left|\int_{-L_{A} / 2}^{+L_{A} / 2} \cos ^{2}\left(\frac{\pi z}{L_{A}}\right) \frac{\mathrm{e}^{+\mathbf{q}_{0} z}+\mathrm{e}^{-\mathbf{q}_{0} z}}{\mathrm{e}^{+\mathbf{q}_{0} L_{A} / 2}+\mathrm{e}^{-\mathbf{q}_{0} L_{A} / 2}} \mathrm{~d} z\right|^{2}} \frac{\mathrm{d} \theta}{2} .
\end{aligned}
$$

Note that due to a symmetry of the first electron subband wavefunction, the intrasubband SR by antisymmetric phonons, $W_{\mathrm{IF}}^{\mathrm{A}}$, is equal to zero.

\section{The sum rule and confined electron-bulk phonon scattering rate approximation}

The calculation of the electron-PO phonon SR, according to Eqs. (19) and (20), neglecting the difference in the electron-IF phonon and electron-confined phonon coupling strengths, i.e. assuming $\omega_{\nu}=\omega_{\mathrm{L} A}$, shows that the sum of the confined electron SR by IF and confined phonons is exactly equal to the confined electron SR by the bulk phonons,

$$
W_{\mathrm{IF}}\left(\omega_{\nu}=\omega_{\mathrm{L} A}\right)+W_{\mathrm{C}}=W_{\mathrm{B}},
$$

where the confined electron SR by bulk phonons, according to the DC model [4], is equal to

$$
\begin{aligned}
W_{\mathrm{B}}\left(\mathbf{k}_{i}\right) & =W_{0} F_{0}\left(N_{q}\left(\omega_{\mathrm{L} A}\right) \pm \frac{1}{2}+\frac{1}{2}\right) \frac{4}{L_{A}^{2}} \\
& \times \int_{0}^{2 \pi} \frac{1}{2 \mathbf{q}_{0}} \int_{-L_{A} / 2}^{+L_{A} / 2} \int_{-L_{A} / 2}^{+L_{A} / 2} \cos ^{2}\left(\frac{\pi z}{L_{A}}\right) \cos ^{2}\left(\frac{\pi z^{\prime}}{L_{A}}\right) \\
& \times \exp \left(-\mathbf{q}_{0}\left|z-z^{\prime}\right|\right) \mathrm{d} z \mathrm{~d} z^{\prime} \frac{\mathrm{d} \theta}{2}
\end{aligned}
$$

where

$$
F_{0}=\frac{\omega_{\mathrm{L} A}^{2}-\omega_{\mathrm{T} A}^{2}}{2 \omega_{\mathrm{L} A} \chi_{\infty A}}, \quad \chi_{\infty A}=\frac{\varepsilon_{\infty A}}{\varepsilon_{0}},
$$

and $\omega_{\mathrm{L} A}$ is expressed in meV.

This fact is adequate to the sum rule of the electronphonon interaction form factors $[4,15]$ and means that, in spite of the PO phonon confinement in the QW, the confined electron scattering by the bulk phonons can be used as an approximation for calculations of the electron-PO phonon SR in the QW. The electron SR by the bulk phonons in the QW is a widely used approximation.

However, the sum rule can not be fulfilled when the IF and confined phonon frequencies, and, therefore, electron interaction strengths with IF and confined phonons are different $[4,15]$. The use of the bulk phonon approximation for calculations of the SR in those cases can give faulty results.

Below, the SR calculations using Eqs. (17)-(19) are compared with the SR calculations within the bulk phonon approximation, Eqs. (21) and (22).

\section{Dependence of electron-IF phonon scattering rate on the $\mathrm{QW}$ width}

Let us consider the electron SR by confined and symmetric IF phonons in three types of double barrier QWs: AlAs / GaAs / AlAs, GaAs / InAs / GaAs, and $\mathrm{GaN} / \mathrm{InN} / \mathrm{GaN}$. The PO phonon parameters of these semiconductors used in calculations are presented in Table 1.

Symmetric IF phonons of a double heterojunction structure (according to Eq. (13)) have two different frequencies: the barrier-like, $\omega_{B}$, and the QW-like, $\omega_{A}$. That means that the SR of electrons with emission of IF phonons has two energy thresholds: at the barrierphonon energy $\hbar \omega_{B}$ and at the QW-phonon energy $\hbar \omega_{A}$. 
Table 1. The PO phonon parameters

\begin{tabular}{ccccc}
\hline & $\omega_{\mathrm{L}}, \mathrm{meV}$ & $\omega_{\mathrm{T}}, \mathrm{meV}$ & $\chi_{\infty}=\varepsilon_{\infty} / \varepsilon_{0}$ & $\mathrm{k}_{\mathrm{opt}}, \mathrm{nm}^{-1}$ \\
\hline $\mathrm{InAs}$ & 30.2 & 27.1 & 12.3 & 0.043 \\
$\mathrm{GaAs}$ & 36.2 & 33.4 & 10.9 & 0.125 \\
$\mathrm{AlAs}$ & 50.1 & 44.8 & 8.16 & \\
$\mathrm{InN}$ & 73 & 48.9 & 6.2 & 0.075 \\
$\mathrm{GaN}$ & 92 & 71.3 & 5.35 & \\
\hline
\end{tabular}

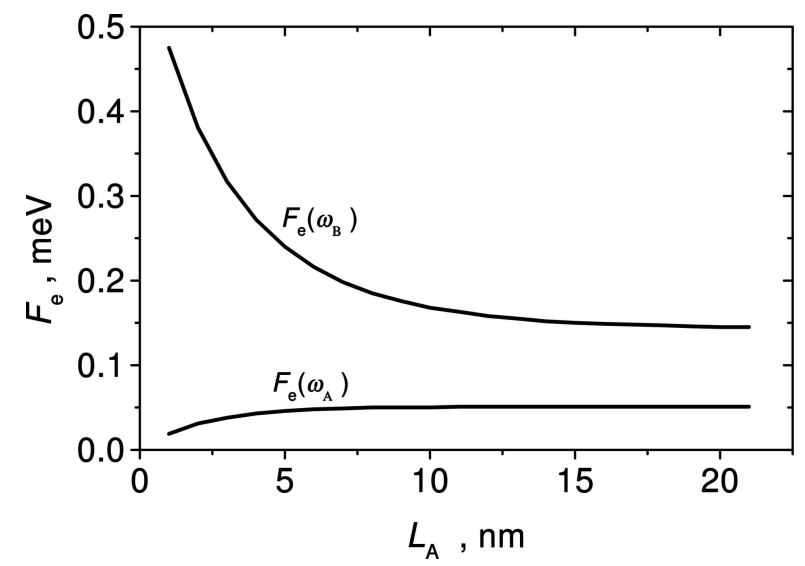

Fig. 3. The QW width dependences of the factor $F_{e}$ (Eq. (23)), which characterizes the electron-phonon interaction strength in the AlAs / GaAs / AlAs double heterostructure. $F_{e}\left(\omega_{B}\right)$ and $F_{e}\left(\omega_{A}\right)$ correspond to the barrier-like and the QW-like frequencies dependent on a QW width.

We calculate the electron-PO phonon SR for three electron energies: (i) the electron energy higher than the barrier-like IF phonon energy; (ii) the electron energy higher than the QW-like phonon energy, but lower than the barrier-like phonon energy; and (iii) the electron energy of $25 \mathrm{meV}$, which is lower than any PO phonon energies considered.

The IF phonon frequency determines the phonon potential amplitude (7) and the electron-phonon interaction strength. This strength we will define as

$$
F_{e}\left(\omega_{\nu}\right)=\frac{1}{\varepsilon_{A}^{\prime}\left(\omega_{\nu}\right) \tanh \left(\frac{\mathbf{q} L_{A}}{2}\right)+\varepsilon_{B}^{\prime}\left(\omega_{\nu}\right)} \varepsilon_{0},
$$

where

$$
\varepsilon_{A}^{\prime}\left(\omega_{\nu}\right)=\frac{2 \omega_{\nu}\left(\omega_{\mathrm{L} A}^{2}-\omega_{\mathrm{T} A}^{2}\right)}{\left(\omega_{\nu}^{2}-\omega_{\mathrm{T} A}^{2}\right)^{2}} \varepsilon_{\infty A}
$$

and

$$
\varepsilon_{B}^{\prime}\left(\omega_{\nu}\right)=\frac{2 \omega_{\nu}\left(\omega_{\mathrm{L} B}^{2}-\omega_{\mathrm{T} B}^{2}\right)}{\left(\omega_{\nu}^{2}-\omega_{\mathrm{T} B}^{2}\right)^{2}} \varepsilon_{\infty B} .
$$

Figure 3 demonstrates the QW width dependence of the factors $F_{e}$ for electron interaction with the barrierlike and the QW-like IF phonons in AlAs / GaAs / AlAs heterostructure.

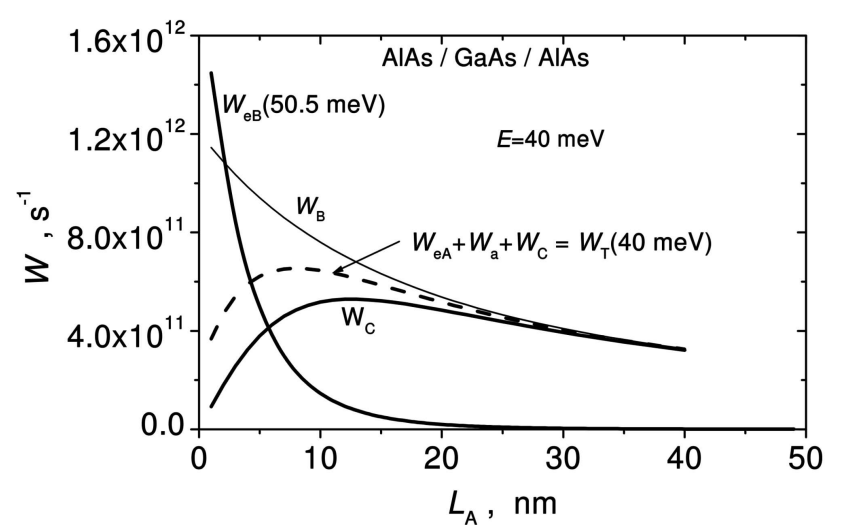

Fig. 4. The QW width dependences of the total SR of the electrons, the energy of which is $40 \mathrm{meV}, W_{\mathrm{T}}(40 \mathrm{meV})$. The partial components of the electron SR are: $W_{\mathrm{e} A}$ is the SR with emission of the QW-like IF phonon, $W_{\mathrm{a}}$ is the SR with absorption of the IF phonon, $W_{\mathrm{C}}$ is the SR with the confined phonon. $W_{\mathrm{B}}$ is the SR calculated within the bulk phonon approximation (Eq. (22)). $W_{\mathrm{e} A}(50.5 \mathrm{meV})$ is the SR of electron, the energy of which is $50.5 \mathrm{meV}$, with emission of the AlAs-like phonon.

It is worth to note that the QW-like symmetric IF mode frequency $\omega_{A}$ approaches $\omega_{\mathrm{T} A}$ limit at $L_{A} \rightarrow 0$, and the interaction strength $F_{e}\left(\omega_{A}\right)$ for the QW-like IF phonons drops to zero (see Eq. (23)). The interaction strength factor for the barrier-like IF mode, $F_{e}\left(\omega_{B}\right)$, monotonously increases with decreasing $L_{A}$.

Thus, in narrow QWs, the electron interaction strength with the high-energy barrier-like phonons increases, and contrary, the electron interaction strength with the QW-like phonons decreases with decreasing $L_{A}$.

An electron, the energy of which is lower than the energy of the high-energy barrier-like IF phonon, cannot emit this phonon, and the SR of the low-energy electron by the IF phonon must decrease due to the strong decrease of the electron interaction strength with the QW-like phonons as compared with the electron interaction strength with the bulk phonons. This is illustrated in Fig. 4.

Figure 4 shows the calculated total SRs of the electrons, the energies of which are 50.5 and $40 \mathrm{meV}$, by the PO phonons as functions of the AlAs / GaAs / AlAs QW width. For comparison, the SR calculated in the bulk phonon approximation is presented.

The IF phonon SR of the electrons, the energy of which is $50.5 \mathrm{meV}$, in the narrow QW is higher than that obtained using the bulk phonon approximation. The increase of factor $F_{e}\left(\omega_{B}\right)$ for the AlAs-like phonons is responsible for this increase.

In the narrow QW, the IF phonon SR of the electrons, the energy of which is $40 \mathrm{meV}$, is much lower than that obtained from the bulk phonon approxima- 


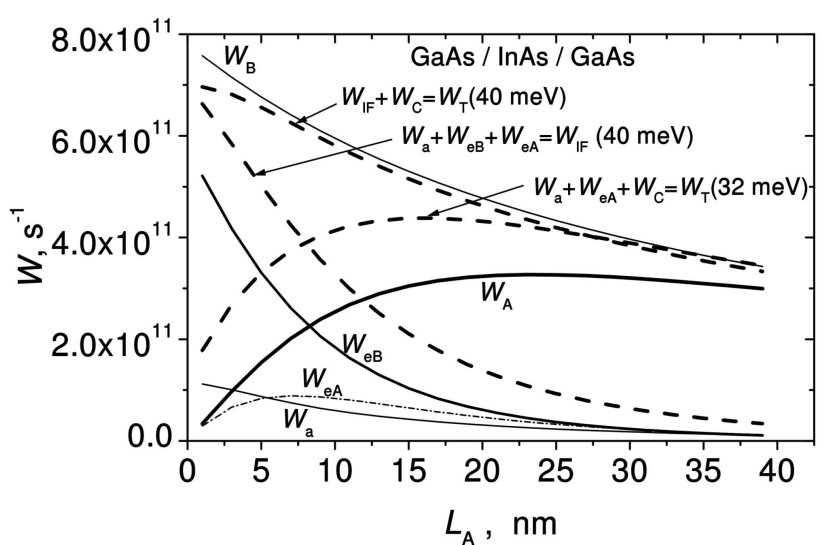

Fig. 5. The QW width dependences of the total SR of electrons, the energies of which are $40 \mathrm{meV}, W_{\mathrm{T}}(40 \mathrm{meV})$, and $32 \mathrm{meV}, W_{\mathrm{T}}(32 \mathrm{meV})$ in $\mathrm{GaAs} / \mathrm{InAs} / \mathrm{GaAs}$. Other notations are as in Fig. 4.

tion. For the electron-IF phonon SR decrease, the $F_{e}\left(\omega_{A}\right)$ decrease is responsible.

The electron-confined phonon SR decreases greatly when the QW width $L_{A}$ becomes less than $L_{\mathrm{opt}}=$ $\pi / k_{\mathrm{opt}}$. In GaAs, $L_{\mathrm{opt}}=12.6 \mathrm{~nm}$. For this decrease, the great increase of the confined phonon wave vector $\mathbf{q}_{\mathrm{C}}=\mathbf{q}+\mathbf{q}_{z}$ is responsible in the narrow QW (see Eqs. (10) and (19)).

Because of the decrease of both confined and IF phonon SRs, the total SR of electrons, the energy of which is $40 \mathrm{meV}$, in the narrow $\mathrm{QW}$ is lower than in the wide one. It can be assumed that this electronPO phonon SR decrease is responsible for the great drift velocity increase observed experimentally in narrow AlAs / GaAs / AlAs QWs [2, 16].

Figure 5 shows the SR dependences on $L_{A}$ in the GaAs / InAs / GaAs QW. As well as in the AlAs / GaAs / AlAs case, the total SR of electrons, the energy of which is lower than the barrier-like IF phonon energy $\left(\hbar \omega_{B}=36 \mathrm{meV}\right)$, decreases with a decrease of the QW width less than $20 \mathrm{~nm}$. As a result, the decrease of the electron-confined phonon SR is not compensated by the IF phonon SR.

At $L_{A} \rightarrow 0$, the total SR is four times lower than the SR given by the bulk phonon approximation. The electron-IF phonon SR by phonon emission decreases due to the change of the QW-like frequency $\omega_{A}$. When $\omega_{A} \rightarrow \omega_{\mathrm{T} A}$, the factor $F_{e}\left(\omega_{A}\right)$ decreases, as well as it was in the AlAs / GaAs / AlAs structure.

However, the SR with phonon emission of the electrons, the energy of which is higher than the energy of the barrier-like phonon, increases with a decrease of the QW width. As a result, the SR of the electrons, the energy of which is $40 \mathrm{meV}$, is near to that $\mathrm{SR}$, which is given by the bulk phonon approximation.

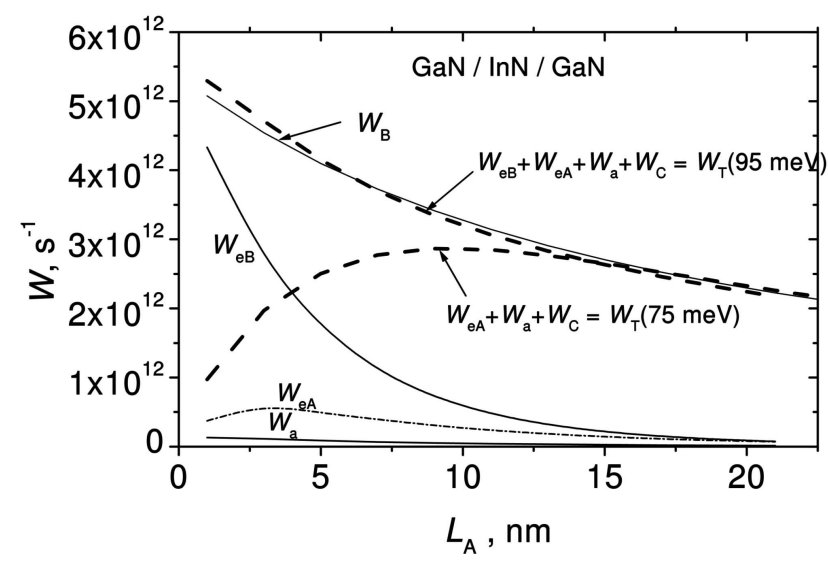

Fig. 6. The QW width dependences of the total SR of electrons, the energies of which are $95 \mathrm{meV}, W_{\mathrm{T}}(95 \mathrm{meV})$, and $75 \mathrm{meV}$, $W_{\mathrm{T}}(75 \mathrm{meV})$ in $\mathrm{GaN} / \mathrm{InN} / \mathrm{GaN}$. Other notations are as in Fig. 4.

Analogical peculiarities in the electron-PO phonon SRs are also observed in $\mathrm{GaN} / \mathrm{InN} / \mathrm{GaN}$ case (Fig. 6): the strong decrease of the total SR of the electrons, the energy of which is lower than $92 \mathrm{meV}$, and the increase of the SR of the higher energy $(95 \mathrm{meV})$ electrons by the highest energy phonons.

Note, that the barrier-like phonon determines the high-energy electron SR. The SR with barrier-like phonon emission in the QW with the GaN barriers (Fig. 6) is more than three times larger than with the AlAs or GaAs barriers (Fig. 4).

The increase of the inelastic SR of hot electrons, the energy of which is higher than the barrier-like phonon energy, shows that the SR can be responsible for the electron drift velocity saturation at high electric fields. Because the barrier-like phonon energy is higher than the bulk-phonon energy, the electron saturated drift velocity in the narrow QW must be higher. The decrease of the SR of the electrons, the energy of which is lower than the highest barrier-like phonon energy, by the PO phonons in the narrow QW means the increase of cold electron mobility in the QW with a width $L_{A}<5 \mathrm{~nm}$.

\section{Conclusions}

The possibilities to decrease the electron-PO phonon SR in the double heterobarrier QW and to increase the electron saturated drift velocity at high electric fields are considered.

It is shown, as an example of three double barrier heterostructures AlAs / GaAs / AlAs, GaAs / InAs / $\mathrm{GaAs}$, and $\mathrm{GaN} / \mathrm{InN} / \mathrm{GaN}$, that these possibilities are realized in narrow QWs $\left(L_{A}<5-10 \mathrm{~nm}\right)$ : 
1. The electron-confined phonon SR decreases due to the strong increase of the confined phonon wave vector in the narrow $\mathrm{QW}$.

2. The IF phonon SR of the electrons, the energy of which is lower than the barrier-like phonon energy, decreases due to the strong decrease of the electron-QW-like phonon interaction strength (factor $F_{e}\left(\omega_{A}\right)$, Eq. (23)) in the narrow QW.

Because of that the mobility of cold electrons limited by PO phonon scattering in the narrow QW can be found higher than in the wide one.

3. The IF phonon SR of the electrons, the energy of which is higher than the barrier-like phonon energy, increases with a QW width decrease due to the increase of the electron-barrier-like phonon interaction (factor $F_{e}\left(\omega_{B}\right)$, Eq. (23)) in the narrow QW.

Because the barrier-like phonon energy is higher than the bulk-like phonon energy, the hot-electron saturated drift velocity in the narrow QW must be higher.

The DC model was used for the SR calculations. The sum rule for the electron SR by the confined and the IF phonons in the QW is well fulfilled, if the difference in the all-mode phonon energies $\hbar \omega_{\nu}$ could be neglected. However, due to the large differences in the energies of confined and IF phonon modes in the narrow $\mathrm{QW}$, the sum rule is violated.

It is shown that the bulk phonon approximation for the electrons, the energy of which is lower than the barrier-like phonon energy, is not fulfilled and cannot be used for the electron-PO phonon SR calculations in the narrow QW.

\section{References}

[1] J. Požela, K. Požela, and V. Jucienè, Enhancement of electron saturated drift velocity in a quantum well by confinement of polar optical phonons, Lithuanian J. Phys. 45, 445-451 (2005).

[2] J.K. Požela and V.G. Mokerov, A large enhancement of the maximum drift velocity of electrons in the channel of a field-effect heterotransistor, Semiconductors 40, 357-361 (2006), Fiz. Tekh. Poluprovodn. 40, 362-366 (2006).
[3] J. Požela, V. Jucienè, A. Namajūnas, and K. Požela, Electron-polar optical phonon scattering rates in modulation-doped AlGaAs/GaAs/AlGaAs double heterojunction structures, Lithuanian J. Phys. 37, 433439 (1997).

[4] N. Mori and T. Ando, Electron-optical phonon interaction in single and double heterostructures, Phys. Rev. B 40, 6175-6188 (1989).

[5] I. Lee, S.M. Goodnick, M. Gulia, E. Molinari, and P. Lugli, Microscopic calculation of the electron-optical phonon interaction in ultrathin $\mathrm{GaAs} / \mathrm{Al}_{x} \mathrm{Ga}_{1-x} \mathrm{As}$ alloy quantum-well systems, Phys. Rev. B 51, 7046-7057 (1995).

[6] C.R. Bennett, M.A. Amato, N.A. Zakhleniuk, B.K. Ridley, and M. Babiker, Effects of monolayer on the electron-phonon scattering rates in a quantum well: Dielectric continuum versus hybrid model, J. Appl. Phys. 45, 1499-1506 (1998).

[7] H. Rücker, E. Molinari, and P. Lugli, Microscopic calculation of the electron-phonon interaction in quantum wells, Phys. Rev. B 45, 6747-6756 (1992).

[8] A.J. Shields, M.P. Chamberlain, M. Cardona, and K. Eberl, Raman scattering due to interface optical phonons in GaAs / AlAs multiple quantum wells, Phys. Rev. B 51, 17728-17739 (1995).

[9] P. Yu and M. Cardona, Fundamentals of Semiconductors (Fiziko-matematicheskaya Literatura, Moscow, 2002) [in Russian].

[10] K.T. Tsen, K.R. Wald, T. Ruf, P.Y. Yu, and H. Morkoc, Electron-optical phonon interactions in ultrathin multiple quantum wells, Phys. Rev. Lett. 67, 2557-2560 (1991).

[11] J. Požela, V. Jucienė, and K. Požela, Electron scattering in quantum well by interface phonons, Lithuanian J. Phys. 36, 149-158 (1996).

[12] B.K. Ridley, Electrons and Phonons in Semiconductor Multilayers (Cambridge University Press, 1997).

[13] K. Huang and B. Zhu, Dielectric continuum model and Fröhlich interaction in superlattices, Phys. Rev. B 38, 13377-13386 (1988).

[14] S. Rudin and T. Reinecke, Electron-LO-phonon scattering rates in semiconductor quantum wells, Phys. Rev. B 41, 7713-7717 (1991).

[15] L.F. Register, Microscopic basis for a sum rule for polar-optical-phonon scattering of carriers in heterostructures, Phys. Rev. B 45, 8756-8759 (1992).

[16] V. Mokerov, J. Požela, K. Požela, and V. Jucienè, A quantum-dot heterostructure transistor with enhanced maximum drift velocity of electrons, Semiconductors 40, 362-366 (2006), Fiz. Tekh. Poluprovodn. 40, 367371 (2006). 


\title{
ELEKTRONU SKLAIDA PAVIRŠINIAIS POLINIAIS OPTINIAIS FONONAIS DVIBARJERIUOSE IVAIRIALYČIUOSE DARINIUOSE
}

\author{
J. Požela, K. Požela, V. Jucienè \\ Puslaidininkiu fizikos institutas, Vilnius, Lietuva
}

\begin{abstract}
Santrauka
Dielektrinio kontinuumo artinyje nagrinėjama suspraustų elektronų sklaida paviršiniais poliniais optiniais fononais dvibarjeriuose ivairialyčiuose dariniuose. Apskaičiuotos elektronų sklaidos paviršiniais fononais spartos priklausomybès nuo kvantinès duobès (KD) pločio ir nuo paviršinių fononų dažnio. Ivertinta KD suspraustų elektronų sklaidos paviršiniais fononais sparta pajuostejje AlAs / GaAs / AlAs, GaAs / InAs / GaAs ir GaN / InN/GaN ịvairialyčiuose dariniuose. Elektronų su energija, didesne už barjero fonono energiją, sklaidos sparta didejja, didejjant šiai fonono energijai. Parodyta, kad elektronų su tūrinių fononų energija KD medžiagoje sklaidos simetriniais paviršiniais fononais sparta labai mažèja siaurejant KD pločiui, kai pastarasis tampa mažesnis už 5-10 nm.
\end{abstract}

Priešingai, elektronų su energija, didesne už didžiausią paviršinio fonono energiją, sklaidos sparta paviršiniais fononais siauroje KD didèja. Tai reiškia, kad elektronu judris ir soties dreifinis greitis stipriuose elektriniuose laukuose siauroje KD turi būti didesni negu plačioje.

Parodyta, kad sumos taisyklè elektronų sklaidos suspraustais ir paviršiniais fononais spartai KD yra patenkinama, jei nepaisoma fononų energijų skirtumų. Tačiau, jei atsižvelgiama i dideli suspraustų ir paviršinių fononų energijų skirtumą siauroje KD, sumos taisyklè negalioja ir tūrinių fononų artinys elektronams su energija, mažesne už barjero fononų energiją, negali būti naudojamas elektronų sklaidos poliniais optiniais fononais skaičiavimuose siauroje KD. 